\title{
SASAKIAN MANIFOLDS, HODGE DECOMPOSITION AND MILNOR ALGEBRAS
}

\author{
Mitsuhiro ITOH \\ (Received 25 April 2003)
}

\begin{abstract}
In this paper we show that Hodge numbers $h^{p, q}$ of a compact Sasakian $(2 n+1)$-manifold $M, 2 n+1 \geq 5$, are given by the dimension of certain linear subspaces of the Milnor algebras $\mathbb{C}\left[z_{i}\right] /\left(\partial f / \partial z_{i}\right)$, when $M$ is a link of an isolated singularity associated with a weighted homogeneous polynomial $f=$ $f\left(z_{1}, \ldots, z_{n+2}\right)$.
\end{abstract}

\section{Introduction}

1.1 .

Let $f=f(z)$ be a polynomial in $z=\left(z_{1}, \ldots, z_{n+2}\right)$ of $\mathbb{C}^{n+2}, n \geq 2$. $f=f(z)$ is said to be weighted homogeneous, of degree $d$ and with weight $\mathbf{w}=\left(w_{1}, \ldots, w_{n+2}\right)$, $w_{i} \in \mathbb{N}, i=1, \ldots, n+2$, if it is a linear combination of monomials $z_{1}^{a_{1}} z_{2}^{a_{2}} \cdots z_{n+2}^{a_{n+2}}$ satisfying $w_{1} a_{1}+\cdots+w_{n+2} a_{n+2}=d$.

The zero locus of $f=f(z), V_{f}=\left\{z \in \mathbb{C}^{n+2} \mid f(z)=0\right\}$, which is an algebraic variety, admits in general the origin as a singularity.

Assume the origin is an isolated singularity so that we intersect $V_{f}$ with a small hypersphere $S_{\varepsilon}^{2 n+3}$ centered at the origin to obtain a compact smooth $(2 n+1)$-manifold

$$
K_{f}=V_{f} \cap S_{\varepsilon}^{2 n+3},
$$

called a link of the singularity. The link $K_{f}$ is equipped with a Sasakian structure. Furthermore, the $\mathbb{C}^{\times}$-action on $\mathbb{C}^{n+2},(t, z) \mapsto t \cdot z=\left(t^{w_{1}} z_{1}, \ldots, t^{w_{n+2}} z_{n+2}\right)$, induces an $S^{1}$-action of contact-isometries on $K_{f}$. This $S^{1}$-action is almost free, namely, it is free but admits finitely many distinct isotropy subgroups contained in some finite subgroup $\Gamma$ of $S^{1}$, so that $K_{f}$ admits a double fibration over a complex $V$-manifold, a complex hypersurface in the weighted projective space $\mathbb{P}^{n+1}(\mathbf{w})$,

$$
\pi: K_{f} \longrightarrow K_{f} / \Gamma \longrightarrow K_{f} / S^{1}=\left(V_{f} \backslash\{0\}\right) / \mathbb{C}^{\times}
$$


The purpose of this paper is to give an explicit formula for the Hodge numbers of a compact Sasakian manifold associated with a weighted homogeneous polynomial.

Even the study of weighted homogeneous polynomials is an interesting subject in mathematical physics, as it is associated with Frobenius algebras and Frobenius manifolds, given by the work of Dubrovin [7]; we will concentrate ourselves on investigating a link of the singularity and the Hodge decomposition structure inherent in the link.

Consider the Hodge decomposition theorem for harmonic forms on the link $K_{f}$. So, recall briefly the harmonic theory of a compact Sasakian manifold established by Tanaka. In his lecture note [23] he discussed it over a normal strongly pseudoconvex CR manifold. However, a normal strongly pseudo-convex CR structure in CR geometry is equivalent to the notion of Sasakian structure in contact geometry, as we will see in Theorem 2.2.

Let $\psi$ be a harmonic $k$-form on such a $(2 n+1)$-manifold $M, k \leq n$. Then it satisfies $i(\xi) \psi=0$, so it is in $\Gamma\left(\Lambda^{k}(E)\right)=\Gamma\left(M ; \Lambda^{k}(E)\right)$ for the contact bundle $E=\operatorname{Ker} \eta=\{X \in T M \mid \eta(X)=0\} \subset T M$.

We split $\Lambda^{k}\left(E_{\mathbb{C}}\right)$ as $\Lambda^{k}\left(E_{\mathbb{C}}\right)=\bigoplus_{p+q=k} \Lambda^{p, q}(E)$ for $E_{\mathbb{C}}=E \otimes \mathbb{C}$ and define the so-called contact Dolbeault operator $\bar{\partial}: \Gamma\left(\Lambda^{p, q}(E)\right) \longrightarrow \Gamma\left(\Lambda^{p, q+1}(E)\right)$, introduced in [23], so that we have the Hodge decomposition theorem as follows.

THEOREM 1.1. (Tanaka [23]) Let $\mathbb{H}^{k}(M)$ be the space of harmonic $k$-forms on a compact Sasakian $(2 n+1)$-manifold. Then, for $k \leq n$,

$$
\mathbb{H}^{k}(M)=\bigoplus_{p+q=k} \mathbb{H}^{p, q}(M), \quad \overline{\mathbb{H}}^{q, p}(M)=\mathbb{H}^{p, q}(M)
$$

in terms of the space $\mathbb{H}^{p, q}(M)$ of $\bar{\partial}$-harmonic $(p, q)$-forms on $M$.

To give the explicit formula of Hodge number $h^{p, q}=\operatorname{dim}_{\mathbb{C}} \mathbb{H}^{p, q}$ for the link $K_{f}$ of the singularity we take account of the double fibration of $K_{f}$ over $V_{f}^{*}=\left(V_{f} \backslash\{0\}\right) / \mathbb{C}^{\times}$and the mixed Hodge structure associated with the Hodge decomposition on $V_{f}^{*}$. Then the Gysin sequence for $K_{f} / \Gamma \longrightarrow V_{f}^{*}$ yields that $h^{p, q}=\operatorname{dim}_{\mathbb{C}} \mathbb{H}_{0}^{p, q}\left(V_{f}^{*}\right)$, as in Theorem 5.3. Here $\mathbb{H}_{0}^{p, q}\left(V_{f}^{*}\right)$ is the space of primitive harmonic $(p, q)$-forms on $V_{f}^{*}$ and is representable from works of Steenbrink in [21], by using the Milnor algebra. Here the Milnor algebra $\mathbb{M}_{f}$ is the quotient of the polynomial ring $\mathbb{C}\left[z_{1}, \ldots, z_{n+2}\right]$;

$$
\mathbb{M}_{f}=\mathbb{C}\left[z_{1}, \ldots, z_{n+2}\right] /\left(\frac{\partial f}{\partial z_{1}}, \ldots, \frac{\partial f}{\partial z_{n+2}}\right)
$$


with degree $\operatorname{deg}\left(z_{i}\right)=w_{i}, \quad i=1, \ldots, n+2$, whose dimension as a vector space is $\left(w_{1}-1\right) \cdots\left(w_{n+2}-1\right)$.

THEOREM 1.2. Let $K_{f}$ be the link of the singularity associated with a weighted homogeneous polynomial $f=f(z)$ in $(n+2)$-complex variables $z$. Then, for $(p, q)$, $p+q=n$,

$$
h^{p, q}\left(K_{f}\right)=\operatorname{dim}_{\mathbb{C}}\left(\mathbb{M}_{f}\right)_{\ell}, \quad \ell=(p+1) d-\sum_{i} w_{i}
$$

where $\left(\mathbb{M}_{f}\right)_{\ell}$ denotes the linear subspace of $\mathbb{M}_{f}$ consisting of degree $\ell$ elements.

From the theory of Milnor fibration [15] the link $K_{f}$ is $(n-1)$-connected so that the only non-trivial Betti numbers are the middle ones, $b_{n}=b_{n+1}$. Therefore, $h^{p, q}\left(K_{f}\right)=0$ for $p+q \neq n, n+1$.

Furthermore, the middle Betti numbers have the following arithmetic formula $[16,18]$ :

$$
b_{n}\left(K_{f}\right)=\sum_{s=0}^{n+2} \sum_{I}(-1)^{n-s} \frac{q_{i_{1}} \cdots q_{i_{s}}}{\left[u_{i_{1}}, \ldots, u_{i_{s}}\right]} .
$$

Here, $I=\left(i_{1}, \ldots, i_{s}\right)$ is an $s$-tuple of indices $1 \leq i_{1}<\cdots<i_{s} \leq n+2$ and $\left[u_{i_{1}}, \ldots, u_{i_{s}}\right]$ denotes the least common multiple of integers $u_{i_{1}}, \ldots, u_{i_{s}}$ (where integers $q_{i}$ and $u_{i}$ are defined as $q_{i}=d / w_{i}$ and as $q_{i}=u_{i} / v_{i}$ with $\left.\left(u_{i}, v_{i}\right)=1\right)$.

Since $b_{n}=\sum_{p=0}^{n} h^{p, n-p}$, we have the following corollary through the intermediary of the Betti number $b_{n}$.

COROLLARY 1.3. The summation of certain vector space dimensions

$$
\sum_{p=0}^{n} \operatorname{dim}_{\mathbb{C}}\left(\mathbb{M}_{f}\right)_{(p+1) d-\sum_{i} w_{i}}
$$

and the arithmetic formula

$$
\sum_{s=0}^{n+2} \sum_{I}(-1)^{n-s} \frac{q_{i_{1}} \cdots q_{i_{s}}}{\left[u_{i_{1}}, \ldots, u_{i_{s}}\right]}
$$

coincide.

The arithmetic formula asserts that the Betti numbers are completely written in the weights $\mathbf{w}=\left(w_{1}, \ldots, w_{n+2}\right)$ and the degree $d$. Therefore, we pose the following. Problem. Does $h^{p, q}\left(K_{f}\right)$ depend only on the degree $d$ and the weights, at least when $\operatorname{dim} K_{f}=5$ ? 
The motivation of our study is to shed light directly on the Hodge decomposition theorem for a compact Sasakian 5-manifold $M$ and hence on the self-duality on $M$. The self-duality in four-dimensions, which is a significantly important geometrical object, is also valid in five-dimensional contact geometry as one has

$$
\Lambda^{2}(E)=\Lambda_{+}^{2}(E) \oplus \Lambda_{-}^{2}(E),
$$

which yields from Tachibana's theorem the self-duality in harmonic form level [22],

$$
\mathbb{H}^{2}(M)=\mathbb{H}_{+}^{2}(M) \oplus \mathbb{H}_{-}^{2}(M) .
$$

One additional remark is that, similarly to the self-duality for a metric (or conformal structure) in four-dimensions, we can say a contact (or Sasakian) 5-manifold to be selfdual relative to the Weyl curvature tensor [10]. On such a 5-manifold we define a CR twistor space over $M$, which is a CR analogue of the Penrose twistor space $[11,12]$. Contact metric (or Sasakian) manifolds are equipped with a much finer structure, an almost complex structure $\left.\varphi\right|_{E}$ on $E$, so one has the splitting $E_{\mathbb{C}}=E^{1,0} \oplus E^{0,1}$ and hence $\Lambda^{2}\left(E_{\mathbb{C}}\right)=\left(\Lambda^{2,0} \oplus \Lambda^{0,2} \oplus \Lambda^{1,1}\right)(E)$. Tanaka's decomposition theorem asserts that $\mathbb{H}^{2}(M) \otimes \mathbb{C}=\mathbb{H}^{2,0} \oplus \mathbb{H}^{0,2} \oplus \mathbb{H}^{1,1}$ with $\mathbb{H}_{+}^{2}(M) \otimes \mathbb{C}=\mathbb{H}^{2,0} \oplus \mathbb{H}^{0,2}, \mathbb{H}_{-}^{2}(M) \otimes \mathbb{C}$ $=\mathbb{H}^{1,1}$, so, in general, numerically $b_{+}(M)=2 h^{2,0}(M), b_{-}(M)=h^{1,1}(M)$.

When $M$ is a principal circle bundle over a degree $d$ complex hypersurface $V_{d}^{*}=\left\{[z] \mid \sum_{i} z_{i}^{d}=0\right\} \subset \mathbb{P}^{3}(\mathbb{C})$, namely a Boothby-Wang-Hatakeyama fibration over $V_{d}^{*}, b_{+}(M)$ and $b_{-}(M)$, respectively, coincide with $b_{+}$and $b_{-}$of the base space; the latter are written as degree-three polynomials in $d$, and are given by $b_{+}=\left(d^{3}-6 d^{2}+11 d-3\right) / 3$ and $b_{-}=\left(2 d^{3}-6 d^{2}+7 d-3\right) / 3$ (see [14]). Inspired by these formulae, we focus on the class of Sasakian manifolds of dimension $2 n+1 \geq 5$ associated with weighted homogeneous polynomials, which is fairly wider than the class associated with homogeneous polynomials.

\section{Sasakian structure}

2.1 .

The link $K_{f}$ of the isolated singularity admits as an odd-dimensional manifold a Sasakian structure. In this section we will, therefore, give a brief sketch of the basic theory of Sasakian structure.

Let $M$ be a smooth $(2 n+1)$-manifold. A triple $(\eta, \xi, \varphi)$ consisting of a 1-form $\eta$, a vector field $\xi$ together with a $(1,1)$-tensor $\varphi$ in $M$ is called a contact structure when they satisfy $\eta \wedge(d \eta)^{n} \neq 0, \eta(\xi)=1, d \eta(\xi, X)=0$ and $\varphi^{2}(X)=-X+\eta(X) \xi$. 
A contact structure $(\eta, \xi, \varphi)$ together with a metric $g$ is called contact metric structure if they satisfy further that $g(\varphi(X), \varphi(Y))=g(X, Y)-\eta(X) \eta(Y)$ and $g(X, \varphi(Y))=$ $\frac{1}{2} d \eta(X, Y)$.

Definition 2.1. A contact metric structure $(\eta, \xi, \varphi, g)$ is Sasakian if the almost complex structure $J$ defined on the product $M \times \mathbb{R}$,

$$
J\left(X+a \frac{d}{d t}\right)=\varphi(X)-a \xi+\eta(X) \frac{d}{d t}, \quad X \in T M, a \in \mathbb{R},
$$

is integrable.

A Sasakian structure is an odd-dimensional analogue of a Kähler structure. In fact, as in [2], we have the following theorem.

THEOREM 2.1. A contact metric structure $(\eta, \xi, \varphi, g)$ is Sasakian if and only if

$$
\left(\nabla_{X} \varphi\right)(Y)=g(X, Y) \xi-\eta(Y) X
$$

where $\nabla$ is the Levi-Civita connection of $g$.

Note that on a Sasakian manifold $\mathcal{L}_{\xi} \phi=0$ and hence $\xi$ is a Killing field with unit norm (see [2, Section 5, IV]).

The contact bundle $E=\operatorname{ker} \eta$, a subbundle of $T M$ with the restriction of $\varphi$, becomes a complex Hermitian vector bundle with a Hermitian fiber metric $\left.g\right|_{E}$, the restriction of $g$.

Split the complexified contact bundle $E_{\mathbb{C}}=E \otimes \mathbb{C}$ as $E_{\mathbb{C}}=E^{1,0} \oplus E^{0,1}$ with respect to $\varphi$. Then we have the following.

Definition 2.2. A contact metric structure $(\eta, \xi, \varphi, g)$ is said to be a strongly pseudoconvex $C R$ structure when $\Gamma\left(M ; E^{1,0}\right)$ is closed under the bracket operation $[\cdot, \cdot]$. A strongly pseudo-convex $\mathrm{CR}$ structure is said to be normal when $\xi$ is $\mathrm{CR}$ holomorphic, i.e. $[\xi, X] \in \Gamma\left(M ; E^{1,0}\right)$ for any $X \in \Gamma\left(M ; E^{1,0}\right)$. For normal strongly pseudo-convex CR structures refer to [23] and [26].

The following is a folklore theorem.

THEOREM 2.2. A contact metric structure is Sasakian if and only if it is normal strongly pseudo-convex $C R$.

Proof. Assume that the almost complex structure $J$ on $M \times \mathbb{R}$ is integrable. Then

$$
[U, V] \in \Gamma\left(M \times \mathbb{R} ; T^{1,0}(M \times \mathbb{R})\right)
$$


for any $U, V \in \Gamma\left(M \times \mathbb{R} ; T^{1,0}(M \times \mathbb{R})\right)$. Note that

$$
T^{1,0}(M \times \mathbb{R})=E^{1,0} \oplus \mathbb{C}\left(\xi-\sqrt{-1} \frac{d}{d t}\right)
$$

and set $U=X+(\xi-\sqrt{-1} d / d t), V=Y+(\xi-\sqrt{-1} d / d t), X, Y \in \Gamma\left(M ; E^{1,0}\right)$. Then, from (3), $[X, Y]$ and $[\xi, X]$ are both in $\Gamma\left(M ; E^{1,0}\right)$ so that the structure is a normal strongly pseudo-convex $\mathrm{CR}$. The converse is easily shown.

\subsection{Almost free $S^{1}$-action}

We call a Sasakian structure $(\eta, \xi, \phi, g) S^{1}$-Sasakian when the vector field $\xi$ generates an $S^{1}$-action. Since $\mathcal{L}_{\xi} \eta=0$ and $\mathcal{L}_{\xi} \phi=0$, this $S^{1}$-action consists of isometric contact transformations of $M$. When this $S^{1}$-action is free, it follows from the classical results of Boothby and Wang [4] and Hatakeyama [9] that the Sasakian structure on $M$ arises from a circle bundle with a connection $\eta$ over a complex Hodge manifold $\left(M^{\prime}, J, G\right)$, so that $g=\pi^{*} G+\eta \otimes \eta, \varphi=\tilde{\pi}_{*} \circ J \circ \pi_{*}\left(\pi: M \longrightarrow M^{\prime}\right.$ is the projection over the space $M^{\prime}$ of all $S^{1}$-orbits in $M$ and $\tilde{\pi}_{*}$ is the horizontal lift) and $\xi$ is the vertical vector field induced from the right action. For this argument we need only the fact that every orbit of $\xi$ is a circle and do not need the compactness of $M$ (see the proof of the theorem of Boothby and Wang given in [2] and also in [24]). Note that an $S^{1}$-Sasakian structure with the $S^{1}$-action which is free is obviously regular contact.

On the other hand, we have another notion: almost regular contact metric structure ([25], see also [3, p. 29]). Associated to this notion we give the following definition.

Definition 2.3. The $S^{1}$-action of an $S^{1}$-Sasakian structure is said to be almost free if the action has no fixed points but the finitely many distinct isotropy subgroups are contained in some finite subgroup $\Gamma$ of $S^{1}$.

Thomas obtained the following result [25]. Let $(M, \eta, \xi, \phi, g)$ be a compact Sasakian manifold or, more generally, a compact contact manifold. If it is almost regular, then the field $\xi$ generates an almost free $S^{1}$-action on $M$ and vice versa.

If the $S^{1}$-action of a Sasakian manifold $M$ is almost free, then $M$ admits a double fibration, first by taking the quotient by the finite subgroup $\Gamma$ and then by the free action of $S^{1} / \Gamma \cong S^{1}$ induced from the original $S^{1}$-action [25]:

$$
M \stackrel{\pi_{1}}{\longrightarrow} M / \Gamma \stackrel{\pi_{2}}{\longrightarrow} M / S^{1}=(M / \Gamma) /\left(S^{1} / \Gamma\right) .
$$

The first fibration is a ramified covering and the second is a proper $S^{1}$-fibration, so that we get the projection $\pi: M \rightarrow M / S^{1}$ as the composition $\pi=\pi_{2} \circ \pi_{1}$. 
A link $K_{f}$ of the singularity associated with a weighted homogeneous polynomial $f$ carries the $S^{1}$-Sasakian structure which is almost free, as explained in Lemma 3.1.

Now assume that $\left(M^{2 n+1}, \eta, \xi, \varphi, g\right)$ is an $S^{1}$-Sasakian manifold for which the $S^{1}$-action generated by $\xi$ is almost free. The isotropy subgroup $\Gamma_{x}$ at $x \in M$ is a finite subgroup of $S^{1}$. We can then apply the isotropy invariant slice lemma (see, for example, [5, II]) to the $S^{1}$-orbit through $x$ so that the quotient space $M / S^{1}$ becomes a real $V$-manifold, i.e. an orbifold of dimension $2 n$. In fact, each point $[x] \in M / S^{1}$ has a neighborhood isomorphic to $U / \Gamma_{x}$, where $U=\left\{W \in E_{x} \mid\|W\|<\varepsilon\right\}$ is an $\varepsilon$-ball in $E_{x}$, a slice at $x$. The tangent space $T_{[x]} M / S^{1}$ is then identified with $E_{x}, x \in \pi^{-1}([x])$. For this argument we used the fact that the $S^{1}$-action is contact, so $E_{x}$ is mapped onto $E_{t \cdot x}$ linearly isomorphically by $t \in S^{1}$.

We now define a Riemannian metric $G$ on $M / S^{1}$, by making use of the identification $E_{x} \cong T_{[x]} M / S^{1}: X \mapsto \underline{X}$ :

$$
G_{[x]}(\underline{X}, \underline{Y})=g_{x}(X, Y)
$$

for $\underline{X}, \underline{Y} \in T_{[x]} M / S^{1}$, identified with $X, Y \in E_{x} . G$ is well-defined, since the $S^{1}$-action is isometric. Then $\pi:(M, g) \longrightarrow\left(M / S^{1}, G\right)$ turns out to be a Riemannian submersion.

Moreover, we can define on $M / S^{1}$ an almost complex structure $J$ just like the free $S^{1}$-action case as follows:

$$
J_{[x]}(\underline{X})=\underline{\varphi(X)} .
$$

Therefore, $G$ is $J$-Hermitian, i.e. $G(J \underline{X}, J \underline{Y})=G(\underline{X}, \underline{Y})$, and furthermore the Kähler form $\Omega=\Omega_{G}$ defined by $\Omega(\underline{X}, \underline{Y})=G(J \underline{X}, \underline{Y})$ is a closed 2-form, since the following is obviously seen:

$$
\pi^{*} \Omega=d \eta
$$

Thus we obtain the following.

THEOREM 2.3. If the $S^{1}$-action of an $S^{1}$-Sasakian manifold is almost free, then the quotient space $M / S^{1}$ admits a real $V$-manifold structure with a Riemannian metric $G$ such that $\pi: M \longrightarrow M / S^{1}$ is a Riemannian submersion. Moreover, the space $M / S^{1}$ carries an almost complex structure $J$ such that $G$ is $J$-Hermitian and the Kähler form $\Omega$ is closed. 


\subsection{The Hodge decomposition}

Let $\mathbb{H}^{k}(M)$ be the space of harmonic $k$-forms on a compact Sasakian $(2 n+1)$-manifold $(M, \eta, \xi, \varphi, g)$ :

$$
\mathbb{H}^{k}(M)=\left\{\psi \in \Gamma\left(\Lambda^{k}\left(T M^{*} \otimes \mathbb{C}\right)\right) \mid d \psi=0, d^{*} \psi=0\right\} .
$$

To obtain the Hodge decomposition of $\mathbb{H}^{k}(M)$ we follow Sections 12 and 13 of [23] and give an outline sketch of the detailed argument.

Assume $\operatorname{dim} M=2 n+1 \geq 5$ and consider

$$
\Lambda^{k}\left(E_{\mathbb{C}}\right)=\bigoplus_{p+q=k} \Lambda^{p, q}(E)
$$

$\Lambda^{k}\left(E_{\mathbb{C}}\right)$ and hence $\Lambda^{p, q}(E)$ admits the Hermitian inner product $\langle\cdot, \cdot\rangle_{E_{\mathbb{C}}}$, induced from $\left.g\right|_{E}$ of $E$. We define several operators, as on a Kähler manifold: the star operator $\star_{E}$, $L$, the exterior product with the 2-form $-d \eta$, and $\Lambda$, the formal adjoint of $L$ :

$$
\begin{aligned}
\star_{E}: \Lambda^{k}\left(E_{\mathbb{C}}\right) \longrightarrow \Lambda^{2 n-k}\left(E_{\mathbb{C}}\right), & \phi \wedge \overline{\star_{E} \psi}=\frac{1}{n !}\langle\phi, \psi\rangle_{E}(d \eta)^{n}, \\
L: \Lambda^{k}\left(E_{\mathbb{C}}\right) \longrightarrow \Lambda^{k+2}\left(E_{\mathbb{C}}\right), & L \psi=(-d \eta) \wedge \psi, \\
\Lambda: \Lambda^{k}\left(E_{\mathbb{C}}\right) \longrightarrow \Lambda^{k-2}\left(E_{\mathbb{C}}\right), & \Lambda \psi=(-1)^{k} \star_{E}\left(L\left(\star_{E} \psi\right)\right) .
\end{aligned}
$$

Furthermore, we define

$$
N=\sqrt{-1} \mathcal{L}_{\xi}: \Gamma\left(\Lambda^{k}\left(E_{\mathbb{C}}\right)\right) \longrightarrow \Gamma\left(\Lambda^{k}\left(E_{\mathbb{C}}\right)\right)
$$

with respect to the Lie derivative in the vector field $\xi$. These are real operators. From the normality of the CR structure we see that $[\xi, \Gamma(E)] \subset \Gamma(E)$ and hence $N$ preserves the $(p, q)$-bidegree of form $\psi$ in $\Lambda^{k}\left(E_{\mathbb{C}}\right) . N$ is self-adjoint with respect to the inner product,

$$
\langle N(\phi), \psi\rangle_{E_{\mathbb{C}}}=\langle\phi, N(\psi)\rangle_{E_{\mathbb{C}}}
$$

and $N$ commutes with the operators $\star_{E}, L$ and $\Lambda$.

THEOREM 2.4. (Tachibana [22]) Let $k \leq n$. A harmonic $k$-form $\psi$ on $M$ satisfies:

(i) $i(\xi) \psi=0$, i.e., $\psi \in \Gamma\left(\Lambda^{k}(E)\right)$;

(ii) $\Lambda \psi=0$.

Since $\xi$ is Killing, $N \psi=\sqrt{-1} \mathcal{L}_{\xi} \psi=0$ for a harmonic form $\psi$ so it follows that, if $k \leq n, \mathbb{H}^{k}(M)$ is a linear subspace of $\left\{\psi \in \Gamma\left(M ; \Lambda^{k}(E)\right) \mid \Lambda \psi=0, N \psi=0\right\}$. 
This theorem partially suggests that we focus on the bundles $\Lambda^{k}\left(E_{\mathbb{C}}\right), \Lambda^{p, q}(E)$ and define Dolbeault operators, analogously to complex manifolds.

Consider the exterior derivative $d \psi$ for $\psi \in \Gamma\left(\Lambda^{p, q}(E)\right)$. Then there exist uniquely $\partial \psi \in \Gamma\left(\Lambda^{p+1, q}(E)\right)$ and $\bar{\partial} \psi \in \Gamma\left(\Lambda^{p, q+1}(E)\right)$ such that

$$
d \psi \equiv \partial \psi+\bar{\partial} \psi \quad \text { modulo } \eta .
$$

The reason for this is that if $\left\{\theta^{1}, \ldots, \theta^{n}\right\}$ is a local frame field of $\Lambda^{1,0}(E)$, then from the integrability of the CR structure each $d \theta^{i}$ admits no components in $\bar{\theta}^{j} \wedge \bar{\theta}^{k}$, i.e.

$$
d \theta^{i} \in \Gamma\left(\Lambda^{2,0} \oplus \Lambda^{1,1}\right)(E) \text { modulo } \eta .
$$

Since any $\psi \in \Gamma\left(\Lambda^{p, q}(E)\right)$ is spanned by $\theta^{i_{1}} \wedge \cdots \wedge \theta^{i_{p}} \wedge \bar{\theta}^{j_{1}} \wedge \cdots \wedge \bar{\theta}^{j_{q}}$, the operators $\partial, \bar{\partial}$ are inductively defined,

$$
\begin{aligned}
& \partial: \Gamma\left(\Lambda^{p, q}(E)\right) \longrightarrow \Gamma\left(\Lambda^{p+1, q}(E)\right), \\
& \bar{\partial}: \Gamma\left(\Lambda^{p, q}(E)\right) \longrightarrow \Gamma\left(\Lambda^{p, q+1}(E)\right),
\end{aligned}
$$

so that one has $d \psi \equiv \partial \psi+\bar{\partial} \psi$ modulo $\eta$. Obviously $\bar{\partial} \psi=\overline{\partial \bar{\psi}}$.

In terms of the $L^{2}$-inner product $(\cdot, \cdot)_{E},(\phi, \psi)_{E}=\int_{M}\langle\phi, \psi\rangle_{E}\left(\eta \wedge(d \eta)^{n}\right)$, in the complex vector space $\Gamma\left(\Lambda^{p, q}(E)\right)$, the operators $\bar{\partial}$, $\partial$ have the formal adjoints $\vartheta$ and $\bar{\vartheta}$, respectively; therefore $\vartheta=\bar{\partial}^{*}$ and $\bar{\vartheta}=\partial^{*}$ so that

$$
\begin{aligned}
& \vartheta=-\star_{E} \circ \partial \circ \star_{E}, \\
& \bar{\vartheta}=-\star_{E} \circ \bar{\partial} \circ \star_{E} .
\end{aligned}
$$

LEMMA 2.5. $\left\{\Gamma\left(\Lambda^{p, q}(E)\right), \partial, \bar{\partial}\right\}$ constitutes a double complex in a generalized sense, that is:

(i)

$$
\partial^{2}=\bar{\partial}^{2}=0 \quad \text { and } \quad \partial \bar{\partial}+\bar{\partial} \partial=-\sqrt{-1} L \circ N
$$

(ii)

$$
\vartheta^{2}=\bar{\vartheta}^{2}=0 \text { and } \vartheta \bar{\vartheta}+\bar{\vartheta} \vartheta=\sqrt{-1} \Lambda \circ N
$$

The most crucial identities in Kähler geometry, called the Hodge identities, are $[8,17]$

$$
[\bar{\partial}, \Lambda]=-\sqrt{-1} \bar{\vartheta}, \quad[\partial, \Lambda]=\sqrt{-1} \vartheta
$$

or equivalently

$$
[\vartheta, L]=-\sqrt{-1} \partial, \quad[\bar{\vartheta}, L]=\sqrt{-1} \bar{\partial}
$$

which also hold on a compact Sasakian manifold, as shown in [23]. 
Now we define the complex Laplacians

$$
\square=\vartheta \bar{\partial}+\bar{\partial} \vartheta, \quad \bar{\square}=\bar{\vartheta} \partial+\partial \bar{\vartheta}
$$

on $\Gamma\left(\Lambda^{k}\left(E_{\mathbb{C}}\right)\right)$. Then, the Hodge identities yield the following beautiful relation between complex Laplacians.

LEMMA 2.6. (Tanaka [23])

$$
\square \psi=\bar{\square} \psi+(n-k) N \psi, \quad \psi \in \Gamma\left(\Lambda^{k}\left(E_{\mathbb{C}}\right)\right) .
$$

Define for $\lambda \in \mathbb{R}$

$$
\mathcal{H}_{\bar{\partial}, \lambda}^{p, q}=\left\{\psi \in \Gamma\left(\Lambda^{p, q}(E)\right) \mid \square \psi=0, N \psi=\lambda \psi\right\} .
$$

The space $\mathcal{H}_{\bar{\partial}, \lambda}^{p, q}$ is closely related, when $\lambda=0$, to the space $\mathbb{H}^{k}(M)$. Indeed we follow [23] to define the subspaces

$$
\begin{aligned}
\mathcal{K}_{\Lambda}^{p, q}(M) & =\left\{\psi \in \mathcal{H}_{\frac{\partial}{\partial}, 0}^{p, q}(M) \mid \Lambda \psi=0\right\} \\
& =\left\{\psi \in \Gamma\left(\Lambda^{p, q}(E)\right) \mid \square \psi=0, N \psi=0, \Lambda \psi=0\right\}
\end{aligned}
$$

and

$$
\begin{aligned}
\mathcal{K}_{L}^{p, q}(M) & =\left\{\psi \in \mathcal{H}_{\frac{1}{\partial}, 0}^{p, q}(M) \mid L \psi=0\right\} \\
& =\left\{\psi \in \Gamma\left(\Lambda^{p, q}(E)\right) \mid \square \psi=0, N \psi=0, L \psi=0\right\} .
\end{aligned}
$$

Then

$$
\mathcal{K}_{L}^{p, q}(M)=\sharp_{E} \mathcal{K}_{\Lambda}^{n-p, n-q}(M)
$$

holds for the operator $\sharp_{E}$ defined by $\sharp_{E} \psi=\overline{\star_{E} \psi}$ and we have eventually the Hodge decomposition theorem.

THEOREM 2.7. (Tanaka [23]) Let $(M, \eta, \xi, \phi, g)$ be a compact Sasakian $(2 n+1)$ manifold. Then the space $\mathbb{H}^{k}(M)$ is decomposed as

(i)

$$
\mathbb{H}^{k}(M)=\bigoplus_{p+q=k} \mathcal{K}_{\Lambda}^{p, q}(M), \quad k \leq n
$$

(ii)

$$
\mathbb{H}^{k}(M)=\bigoplus_{p+q=k} \eta \wedge \mathcal{K}_{L}^{p, q}(M), \quad k \geq n+1 .
$$


So, set $\mathbb{H}^{p, q}(M)=\mathcal{K}_{\Lambda}^{p, q}(M)$, when $p+q \leq n$. The above theorem then asserts for $k \leq n$ that $\mathbb{H}^{p, q}(M)$ is the space of harmonic $(p, q)$-forms on $M$ and the space $\mathbb{H}^{k}(M)$ admits the Hodge decomposition:

$$
\begin{aligned}
\mathbb{H}^{k}(M) & =\bigoplus_{p+q=k} \mathbb{H}^{p, q}(M), \\
\overline{\mathbb{H}}^{p, q}(M) & =\mathbb{H}^{q, p}(M), \quad k \leq n
\end{aligned}
$$

Remark. For $k \geq n+1$, set $\mathbb{H}^{p, q}(M)=\eta \wedge \mathcal{K}_{L}^{p-1, q}(M), p+q=k$. Then one has $\mathbb{H}^{k}(M)=\bigoplus_{p+q=k} \mathbb{H}^{p, q}(M)$, but with the property $\overline{\mathbb{H}}^{p, q}(M)=\mathbb{H}^{q+1, p-1}(M)$.

\section{The $S^{1}$-Sasakian structure of $K_{f}$}

\section{1. $\mathbb{C}^{\times}$-action}

Let

$$
\begin{gathered}
\mathbb{C}^{\times} \times \mathbb{C}^{n+2} \longrightarrow \mathbb{C}^{n+2} ; \\
(t, z) \mapsto t \cdot z=\left(t^{w_{1}} z_{1}, \ldots, t^{w_{n+2}} z_{n+2}\right)
\end{gathered}
$$

be the weighted $\mathbb{C}^{\times}$-action on $\mathbb{C}^{n+2}$. Then, the weighted homogeneous polynomial $f$ satisfies $f(t \cdot z)=t^{d} f(z)$ so that the variety $V_{f}$ is $\mathbb{C}^{\times}$-invariant. The link $K_{f}$ of the singularity is also invariant under the action of $S^{1}=\left\{e^{\sqrt{-1} \theta} \mid \theta \in \mathbb{R}\right\}$.

Assume that $\operatorname{GCD}\left\{w_{1}, \ldots, w_{n+2}\right\}=1$ which guarantees effectiveness of the $\mathbb{C}^{\times}$-action on $V_{f}$ and of the $S^{1}$-action on $K_{f}$.

LEMMA 3.1. (Dimca [6]) The isotropy subgroup $\Gamma_{z}, z \in K_{f}$, is the multiplicative group of $w(z)$-roots of unity,

$$
\Gamma_{z}=\left\{\zeta \in S^{1} \mid \zeta^{w(z)}=1\right\}
$$

where $w(z)=\operatorname{GCD}\left\{w_{i} \mid z_{i} \neq 0\right\}$ for $z=\left(z_{1}, \ldots, z_{n+2}\right)$.

The proof is simply arithmetic, so we omit it. From this lemma it follows that the $S^{1}$-action on $K_{f}$ is almost free.

\section{2. $S^{1}$-Sasakian structure on $K_{f}$}

To introduce a Sasakian structure on the link $K_{f}$, we define instead, by virtue of Theorem 2.1, a normal strongly pseudo-convex CR structure on $K_{f}$. 
Let $E^{1,0}$ be the subbundle of $T K_{f} \otimes \mathbb{C}$ defined by

$$
E_{z}^{1,0}=\left\{X \in T_{z}^{1,0} \mathbb{C}^{n+2} \mid X f=0, X \zeta=0\right\}, \quad z \in K_{f}
$$

and $E^{0,1}$ its complex conjugate; $E^{0,1}=\bar{E}^{1,0}$ (where $\zeta=\sum_{i}\left|z_{i}\right|^{2}-\varepsilon^{2}$ is a smooth function defining the sphere $\left.S_{\varepsilon}^{2 n+3}\right)$. We see that $\operatorname{dim}_{\mathbb{C}} E^{1,0}=n$.

It is easily seen that $\Gamma\left(E^{1,0}\right)$ is closed under the bracket operation $[\cdot, \cdot]$. We define the vector field $\xi$ as $\xi_{z}=d /\left.d \theta\left(e^{-\sqrt{-1} \theta} \cdot z\right)\right|_{\theta=0}$, just as the vector field on $\mathbb{C}^{n+2}$ induced from the $S^{1}$-action. Then

$$
\xi=\sqrt{-1} \sum_{i=1}^{n+2} w_{i}\left(z_{i} \frac{\partial}{\partial z_{i}}-\bar{z}_{i} \frac{\partial}{\partial \bar{z}_{i}}\right) .
$$

Since the $S^{1}$-action leaves both $V_{f}$ and $S_{\varepsilon}^{2 n+3}$ invariant, one can see that the restriction of $\xi$ to $K_{f}$ is a vector field, which we denote by the same letter, and that it satisfies

$$
\left[\xi, \Gamma\left(E^{1,0}\right)\right] \subset \Gamma\left(E^{1,0}\right) .
$$

On $K_{f}, \sum_{i}\left(z_{i} d \bar{z}_{i}+\bar{z}_{i} d z_{i}\right)=0$, i.e. $\sum_{i} \bar{z}_{i} d z_{i}=-\sum_{i} z_{i} d \bar{z}_{i}$ holds. So we set a 1-form $\eta$ as

$$
\eta=\sqrt{-1}\left(\sum_{j} w_{j}\left|z_{j}\right|^{2}\right)^{-1} \sum_{i} \bar{z}_{i} d z_{i}
$$

Then we have $\eta(\xi)=1$.

Furthermore, the 2-form $d \eta$, which is represented as

$$
\begin{aligned}
d \eta & =-\frac{\sqrt{-1}}{\left(\sum_{j} w_{j}\left|z_{j}\right|^{2}\right)} \sum_{i} d z_{i} \wedge d \bar{z}_{i} \\
& -\frac{\sqrt{-1}}{\left(\sum_{j} w_{j}\left|z_{j}\right|^{2}\right)^{2}} d\left(\sum_{j} w_{j}\left|z_{j}\right|^{2}\right) \wedge\left(\sum_{i} \bar{z}_{i} d z_{i}\right),
\end{aligned}
$$

reduces to

$$
-\frac{\sqrt{-1}}{\left(\sum_{j} w_{j}\left|z_{j}\right|^{2}\right)} \sum_{i} d z_{i} \wedge d \bar{z}_{i}
$$

when restricted to $E^{1,0}$, since $\left(\sum_{i} \bar{z}_{i} d z_{i}\right)(X)=X \zeta=0$ for $X \in E^{1,0}$. So, the form $d \eta$ yields a positive definite Hermitian form, called the Levi form on $E^{1,0}$ :

$$
\mathbb{L}(X, \bar{Y})=\frac{1}{\left(\sum_{j} w_{j}\left|z_{j}\right|^{2}\right)} \sum_{i=1}^{n+2}\left(X z_{i}\right)\left(\overline{Y z_{i}}\right), \quad X, Y \in E^{1,0} .
$$


One can easily see that $d \eta(\xi, \cdot)=0$.

The direct sum $E^{1,0} \oplus E^{0,1}$ defines a real subbundle $E$ of the real tangent bundle $T K_{f}$ at $z$ of $K_{f}$, such that the complexification of $E$ is

$$
E_{\mathbb{C}}=E^{1,0} \oplus E^{0,1},
$$

together with an endomorphism $\varphi$ of $E$ satisfying $\left.\varphi\right|_{E^{1,0}}=\sqrt{-1}$ id and $\left.\varphi\right|_{E^{0,1}}=$ $-\sqrt{-1}$ id. One then has, at $z$

$$
\mathbb{L}(X, \bar{Y})=d \eta(\varphi(X), \bar{Y}), \quad X, Y \in E_{z}^{1,0},
$$

and one obtains a smooth Riemannian metric $g$ on $K_{f}$ as $g(\xi, \xi)=1$ and $g(\xi, X)=0$ for any $X$ of $E_{z}$ and $2 g(X, Y)=\mathbb{L}\left(X^{1,0}, \bar{Y}^{1,0}\right)+\mathbb{L}\left(Y^{1,0}, \bar{X}^{1,0}\right)$ for real vectors $X=X^{1,0}+\bar{X}^{1,0}, Y=Y^{1,0}+\bar{Y}^{1,0}$, so that $g(X, \varphi Y)=\frac{1}{2} d \eta(X, Y)$.

Therefore, it follows from the theorem of Tanaka (see [23, 11.2, III]) that $(\eta, \xi, \varphi)$ together with the induced metric $g$ yields on $K_{f}$ a normal strongly pseudo-convex CR structure.

Therefore, we are able to summarize the above arguments as follows.

THEOREM 3.2. The link $K_{f}=V_{f} \cap S_{\varepsilon}^{2 n+3}$ associated with a weighted homogeneous singularity carries an $S^{1}$-action and is equipped with a normal strongly pseudo-convex $C R$ structure, namely, a Sasakian structure $(\eta, \xi, \varphi, g)$. This $S^{1}$-action is almost free and consists of isometric contact transformations of the Sasakian structure.

Note that Sasaki and Hsu [19] introduced directly a Sasakian structure on $K_{f}$ for a weighted homogeneous polynomial $f$ of Brieskorn-Pham type.

\subsection{The Hodge structure on $K_{f} / S^{1}$}

The $S^{1}$-action of $K_{f}$ is almost free and so we write the projection $\pi: K_{f} \longrightarrow K_{f} / S^{1}$ as the composition of the fibrations $\pi_{1}: K_{f} \longrightarrow K_{f} / \Gamma$ and $\pi_{2}: K_{f} / \Gamma \longrightarrow K_{f} / S^{1}$. Here $\Gamma$ is a finite subgroup of $S^{1}$ containing all the $\Gamma_{z}, z \in K_{f}$.

The quotient space $K_{f} / S^{1}$ is described as $\left(V_{f} \backslash\{0\}\right) / \mathbb{C}^{\times}$, a complex hypersurface in $\mathbb{P}^{n+1}(\mathbf{w})$. Here the space $\mathbb{P}^{n+1}(\mathbf{w})=\left(\mathbb{C}^{n+2} \backslash\{0\}\right) / \mathbb{C}^{\times}$is the weighted projective space of weight $\mathbf{w}=\left(w_{1}, \ldots, w_{n+2}\right)$, obtained by the weighted $\mathbb{C}^{\times}$-action. We write the weighted projective space as the quotient of the ordinary complex projective space,

$$
\mathbb{P}^{n+1}(\mathbf{w})=\mathbb{P}^{n+1}(\mathbb{C}) / \Gamma(\mathbf{w}),
$$

from which $\mathbb{P}^{n+1}(\mathbf{w})$ is a complex $V$-manifold. Here $\Gamma(\mathbf{w})=\Gamma\left(w_{1}\right) \times \cdots \times \Gamma\left(w_{n+2}\right)$ is the product group of $\Gamma\left(w_{i}\right)=\left\{\zeta \mid \zeta^{w_{i}}=1\right\}, i=1, \ldots, n+2$. 
Denote by $V_{f}^{*}$ the weighted complex hypersurface $K_{f} / S^{1}$.

From Theorem 2.3, the quotient space $V_{f}^{*}=K_{f} / S^{1}$ is a $V$-manifold with an almost Hermitian structure $(J, G)$ so that the projection $\pi: K_{f} \longrightarrow V_{f}^{*}$ is a Riemannian submersion.

We next assert is that the almost Hermitian structure $(J, G)$ which $V_{f}^{*}$ carries is a Hodge structure in the sense of a $V$-manifold, so that the $J$ is the complex structure canonically induced from the affine hypersurface $V_{f} \subset \mathbb{C}^{n+2}$ and hence the differential map $\pi_{*}: E_{z}^{1,0} \longrightarrow T_{[z]}^{1,0} V_{f}^{*}$ is complex linear with respect to $\varphi$ and $J$ at any $z \in K_{f}$.

To assert this we write the holomorphic tangent space $T_{z}^{1,0} V_{f}, z \in K_{f}$,

$$
T_{z}^{1,0} V_{f}=E_{z}^{1,0} \oplus \mathbb{C} \xi^{1,0},
$$

where $\xi^{1,0}$ is the weighted holomorphic Euler vector field, induced from the weighted holomorphic $\mathbb{C}^{\times}$-action,

$$
\xi^{1,0}=\left.\left(\sum_{i} w_{i} z_{i} \frac{\partial}{\partial z_{i}}\right)\right|_{V_{f}} .
$$

The holomorphic tangent space of $V_{f}^{*}$ with respect to the canonically induced complex structure is, by definition, the quotient space $T_{z}^{1,0} V_{f}$ by the $\mathbb{C}$-linear subspace $\mathbb{C} \xi^{1,0}$, and hence coincides with $E_{z}^{1,0}$. Therefore, this implies that the almost complex structure $J$ is exactly the one canonically induced from $V_{f} \subset \mathbb{C}^{n+2}$. Summing up these arguments, we have the following theorem.

THEOREM 3.3. The quotient space $K_{f} / S^{1}=V_{f}^{*}$ is a complex $V$-manifold with a Hodge structure $(J, G)$ such that $J$ is canonically induced from the projection $V_{f} \longrightarrow V_{f}^{*}$ and $\pi$ is a Riemannian submersion and satisfies $\pi^{*} \Omega=d \eta$.

Remarks.

(1) Since $\Gamma(\mathbf{w})$ acts on $\mathbb{P}^{n+1}(\mathbb{C})$ bi-holomorphically and isometrically, $\mathbb{P}^{n+1}(\mathbf{w})$ admits the Fubini-Study metric as a $V$-manifold. However, our metric $G$ defined on $V_{f}^{*}$ does not, in general, coincide with the metric induced from the embedding $\iota: V_{f}^{*} \hookrightarrow \mathbb{P}^{n+1}(\mathbf{w})$.

(2) The complex $V$-manifold structure which $V_{f}^{*}$ carries is dealt by Steenbrink (see [21, Lemma 1], where he treated the case of weighted homogeneous polynomials of special type $\left.f=h\left(z_{1}, \ldots, z_{n+1}\right)+z_{n+2}^{d}\right)$.

The final remark of this section is on the free-action points of $K_{f}$. Denote by $\breve{K}_{f}=\left\{z \in K_{f} \mid G_{z}=e\right\}$ the set of free-action points of $K_{f} . \breve{K}_{f}$ is an open dense 
subset of $K_{f}$. The projection $\pi$ then yields a principal $S^{1}$-bundle when one restricts it to $\breve{K}_{f}$,

$$
\pi: \breve{K}_{f} \longrightarrow \breve{V}_{f}^{*},
$$

over a smooth hypersurface part $\breve{V}_{f}^{*}=\pi\left(\breve{K}_{f}\right) \subset \pi\left(K_{f}\right)$ in $\mathbb{P}^{n+1}(\mathbf{w})$. Even though $\breve{K}_{f}$ is non-compact as remarked in 2.2 , the Sasakian structure $(\eta, \xi, \varphi, g)$ restricted to $\breve{K}_{f}$ is correctly a Boothby-Wang-Hatakeyama fibration of the Hodge structure of $\breve{V}_{f}^{*}$, the restriction of the Hodge structure $(J, G)$ of $V_{f}^{*}$.

We note further that the differential map $\pi_{*}: E_{z} \longrightarrow T_{\pi(z)} V_{f}^{*}$, at any $z \in K_{f}$, yields an isomorphism between $\Lambda^{p, q}(E)$ and the bundle $\Lambda^{p, q}\left(V_{f}^{*}\right)$ of $(p, q)$-forms on $V_{f}^{*}$, even though $V_{f}^{*}$ is a $V$-manifold.

\section{Milnor fibration}

\section{1.}

Following Milnor [15], define the map, called the Milnor fibration,

$$
\Phi: S_{\varepsilon}^{2 n+3} \backslash K_{f} \longrightarrow S^{1} ; \quad z \mapsto f(z) /|f(z)| .
$$

Each fiber $F_{\theta}=\Phi^{-1}\left(e^{\sqrt{-1} 2 \pi \theta}\right) \subset S_{\varepsilon} \backslash K_{f}, \quad e^{\sqrt{-1} 2 \pi \theta} \in S^{1}$, is a smooth, parallelizable $(2 n+2)$-manifold with boundary $\partial F_{\theta}=K_{f}$. Since the origin 0 is an isolated singularity of $V_{f}, K_{f}$ is $(n-1)$-connected and $F_{\theta}$ has the homotopy type of a bouquet of $(n+1)$-spheres. Let $F=F_{1}$ and define $h: F=F_{1} \longrightarrow F=F_{0}$, the monodromy map, so that the product space $F \times[0,2 \pi]$ yields $S_{\varepsilon} \backslash K_{f}$ by identifying $F \times 0$ and $F \times 2 \pi$ by the $h$.

Consider the induced homomorphism

$$
h_{*}: H_{n+1}(F ; \mathbb{C}) \longrightarrow H_{n+1}(F ; \mathbb{C})
$$

of the $(n+1)$ th homology group of $F$. The Wang sequence associated with the Milnor fibration reduces to the short exact sequence

$$
0 \longrightarrow H_{n+1}\left(K_{f}\right) \longrightarrow H_{n+1}(F) \stackrel{I-h_{*}}{\longrightarrow} H_{n+1}(F) \longrightarrow H_{n}\left(K_{f}\right) \longrightarrow 0
$$

and it is asserted that

$$
H_{n+1}\left(K_{f}\right)=\operatorname{Ker}\left(I-h_{*}\right), \quad H_{n}\left(K_{f}\right)=\operatorname{Coker}\left(I-h_{*}\right)
$$

and $H_{n+1}(F)$ is free abelian and of rank $\mu=\left(w_{1}-1\right)\left(w_{2}-1\right) \cdots\left(w_{n+2}-1\right)$. 
Milnor proves further that the fiber $F$ is diffeomorphic to a non-singular variety

$$
F^{\prime}=\left\{z \in \mathbb{C}^{n+2} \mid f(z)=1\right\}
$$

and the $h$ may be chosen as

$$
h^{\prime}: F^{\prime} \longrightarrow F^{\prime}, \quad h^{\prime}(z)=\left(\zeta^{w_{1}} z_{1}, \ldots, \zeta^{w_{n+2}} z_{n+2}\right),
$$

where $\zeta=\exp (2 \pi \sqrt{-1} / d)$. Therefore, the linear transformation $h_{*}^{\prime}: H_{n+1}\left(F^{\prime}\right) \longrightarrow$ $H_{n+1}\left(F^{\prime}\right)$ has finite period and it can be diagonalized by a suitable basis. The multiplicity $k$ of the solution $t=1$ in the characteristic polynomial $\Delta(t)=\operatorname{det}\left(t I-h_{*}^{\prime}\right)$ therefore gives the rank of $H_{n+1}\left(K_{f}\right)$, i.e. the Betti number $b_{n}=b_{n+1}$.

The multiplicity $k$ can be computed by the divisor's argument and Weil's $\zeta$-function argument $[\mathbf{1 6}, \mathbf{1 8}])$. Therefore, one has the following theorem.

THEOREM 4.1. [16, 18]

$$
b_{n}\left(K_{f}\right)=\sum_{s=0}^{n+2} \sum_{I}(-1)^{n-s} \frac{q_{i_{1}} \times \cdots \times q_{i_{s}}}{\left[u_{i_{1}}, \ldots, u_{i_{s}}\right]} .
$$

\section{The main theorem}

5.1 .

The $S^{1}$-action of the Sasakian manifold $K_{f}$ is almost free so that from the argument of Thomas one has the double fibration,

$$
K_{f} \stackrel{\pi_{1}}{\longrightarrow} K_{f} / \Gamma \stackrel{\pi_{2}}{\longrightarrow} V_{f}^{*}=K_{f} / S^{1}=\left(V_{f} \backslash\{0\}\right) / \mathbb{C}^{\times} .
$$

Here $\pi_{1}$ is a branched covering and $\pi_{2}$ is a projection by a free $S^{1} / \Gamma\left(\cong S^{1}\right)$-action. Furthermore, $V_{f}^{*}$ is a complex hypersurface in the weighted projective $(n+1)$-space $\mathbb{P}^{n+1}(\mathbf{w}) . \quad V_{f}^{*}$ is in general a $V$-manifold, as we saw in Section 3. Note that the $\Gamma$-quotient $K_{f} / \Gamma$ is also a real $V$-manifold.

LEMMA 5.1. The nth cohomology group $H^{n}\left(K_{f} / \Gamma ; \mathbb{R}\right)$ is isomorphic to $\mathbb{H}^{n}\left(K_{f}\right)$ of $K_{f}$ through $\pi_{1}$,

$$
H^{n}\left(K_{f} / \Gamma ; \mathbb{R}\right) \cong \mathbb{H}^{n}\left(K_{f}\right)
$$

Proof. Since the space $K_{f} / \Gamma$ is a finite quotient of a smooth manifold, one obtains $H^{n}\left(K_{f} / \Gamma ; \mathbb{R}\right) \cong \mathbb{H}^{n}\left(K_{f}\right)^{\Gamma}$, the space of $\Gamma$-invariant harmonic $n$-forms on $K_{f}$ (see [5, 2.4 Theorem, III]). The $S^{1}$-action is isometric so that any harmonic form on $K_{f}$ is $\Gamma$-invariant. So $\mathbb{H}^{n}\left(K_{f}\right)^{\Gamma}=\mathbb{H}^{n}\left(K_{f}\right)$. 


\subsection{Gysin sequence}

Next consider the $S^{1}$-fibration $\pi_{2}: K_{f} / \Gamma \longrightarrow K_{f} / S^{1}=V_{f}^{*}$ to which we can apply the Gysin exact sequence of the cohomology groups [5],

$$
\begin{aligned}
& \longrightarrow H^{n-1}\left(K_{f} / \Gamma\right) \stackrel{\int}{\longrightarrow} H^{n-2}\left(V_{f}^{*}\right) \stackrel{b}{\longrightarrow} H^{n}\left(V_{f}^{*}\right) \\
& \stackrel{\pi_{2}^{*}}{\longrightarrow} H^{n}\left(K_{f} / \Gamma\right) \stackrel{\int}{\longrightarrow} H^{n-1}\left(V_{f}^{*}\right) \stackrel{b}{\longrightarrow} .
\end{aligned}
$$

Here $b: H^{n-2}\left(V_{f}^{*}\right) \longrightarrow H^{n}\left(V_{f}^{*}\right)$ is the map : $[\alpha] \mapsto[\alpha] \wedge[\Omega]$ where $\Omega$ is the Kähler form on $V_{f}^{*}$, defined from the Hodge structure $(J, G)$ of $V_{f}^{*}$, representing the curvature form of the $S^{1}$-fibration $\pi_{2}$. The map $\int$ is an integrating map along fibers so $\int$ reduces to the zero map, since harmonic forms in $K_{f} / \Gamma$ or in $K_{f}$ have no fiber directional part, i.e. no $\xi$-directional part. See Theorem 2.4. Therefore, the map $b$ is injective and we have

$$
H^{n}\left(V_{f}^{*}\right) \cong H_{0}^{n}\left(V_{f}^{*}\right) \oplus H^{n-2}\left(V_{f}^{*}\right)
$$

Here, $H_{0}^{n}\left(V_{f}^{*}\right)=\left\{[\alpha] \in H^{n}\left(V_{f}^{*}\right) \mid b^{*}[\alpha]=0\right\}$ is the primitive cohomology group of $V_{f}^{*}$, naturally isomorphic to $H^{n}\left(V_{f}^{*}\right) / \operatorname{Im} b$. We therefore get the isomorphism via the Gysin sequence.

LEMMA 5.2.

$$
\pi_{2}^{*}: H_{0}^{n}\left(V_{f}^{*}\right) \stackrel{\cong}{\longrightarrow} H^{n}\left(K_{f} / \Gamma\right)\left(\cong \mathbb{H}^{n}\left(K_{f}\right)\right)
$$

is an isomorphism.

5.3 .

Since $V_{f}^{*}$ is a complex $V$-manifold, the Hodge-de Rham-Kodaira harmonic theory can also be applied to $V_{f}^{*}[\mathbf{1}]$. Therefore, $H_{0}^{n}\left(V_{f}^{*}\right)$, which is regarded as the space $\mathbb{H}_{0}^{n}\left(V_{f}^{*}\right)$ of primitive harmonic forms on $V_{f}^{*}$, splits into the direct sum of the space $\mathbb{H}_{0}^{p, q}\left(V_{f}^{*}\right)$ of primitive harmonic $(p, q)$-forms on $V_{f}^{*}$,

$$
H_{0}^{n}\left(V_{f}^{*}\right) \cong \bigoplus_{p+q=n} \mathbb{H}_{0}^{p, q}\left(V_{f}^{*}\right)
$$

where a harmonic form $\psi$ is primitive provided it satisfies $\Lambda_{\Omega_{G}} \psi=0$, and $\mathbb{H}_{0}^{p, q}\left(V_{f}^{*}\right)=\left\{\bar{\partial}\right.$-harmonic $(p, q)$ form $\psi^{p, q}$ on $\left.V_{f}^{*} \mid \Lambda_{\Omega_{G}} \psi^{p, q}=0\right\}$.

THEOREM 5.3. For non-negative integers $p, q$ with $p+q=n$,

$$
\mathbb{H}^{p, q}\left(K_{f}\right) \cong \mathbb{H}_{0}^{p, q}\left(V_{f}^{*}\right) .
$$


Proof. A primitive harmonic $n$-form $\psi$ on $V_{f}^{*}$ is written as the direct sum of harmonic $(p, q)$-forms, $\psi=\sum_{p+q=n} \psi^{p, q}, \psi^{p, q} \in \mathbb{H}_{0}^{p, q}\left(V_{f}^{*}\right)$. Thus the lift of $\psi$ by $\pi$ is $\pi^{*} \psi=\sum_{p+q=n} \pi^{*} \psi^{p, q}$. From the property of the projection $\pi, \pi^{*} \psi^{p, q}$ is a $(p, q)$-form on $K_{f}$ with no $\xi$-component. Since $\pi$ is a Riemannian submersion with totally geodesic fibers, $\pi^{*} \psi^{p, q}$ is also harmonic in $K_{f}$, even $\pi$ is a submersion onto a $V$-manifold. On the other hand, it is easily seen that $\pi^{*}$ is injective. So, $\operatorname{dim}_{\mathbb{C}} \mathbb{H}_{0}^{p, q}\left(V_{f}^{*}\right) \leq \operatorname{dim}_{\mathbb{C}} \mathbb{H}^{p, q}\left(K_{f}\right)$. Therefore, by counting the dimension of both the cohomology groups $\mathbb{H}_{0}^{n}\left(V_{f}^{*}\right)$ and $\mathbb{H}^{n}\left(K_{f}\right)$, which must be the same from Lemma 5.2, we have the equality in Theorem 5.3.

\subsection{The mixed Hodge numbers}

Hodge-de Rham-Kodaira's theorem and Dolbeault-Kodaira's theorem, which work on a complex $V$-manifold, do not depend on the choice of Kähler metric ([1, Theorems $\mathrm{H}, \mathrm{K}]$ ). So, we can identify $\mathbb{H}_{0}^{p, q}\left(V_{f}^{*}\right)$ with $H^{p, q}\left(V_{f}^{*}\right) / \operatorname{Im} j^{n}$, where $j^{n}: H^{n}\left(\mathbb{P}^{n+1}(\mathbf{w})\right) \longrightarrow H^{n}\left(V_{f}^{*}\right)$ is the monomorphism induced by the inclusion $j: V_{f}^{*} \longrightarrow \mathbb{P}^{n+1}(\mathbf{w})$ (see [6, (B25)Lemma/Definition, Appendix B]). The following key theorem allows us to compute the mixed Hodge numbers $h_{0}^{p, q}\left(V_{f}^{*}\right)=\operatorname{dim}_{\mathbb{C}} H_{0}^{p, q}\left(V_{f}^{*}\right)$ of the primitive cohomology group $H_{0}^{n}\left(V_{f}^{*}\right)$.

THEOREM 5.4. [20, 21]

$$
h_{0}^{p, q}\left(V_{f}^{*}\right)=\operatorname{dim}_{\mathbb{C}}\left(\mathbb{M}_{f}\right)_{\ell},
$$

where $\mathbb{M}_{f}$ is the Milnor algebra associated to the weighted homogeneous polynomial $f$, introduced in Section 1, and $\ell=(p+1) d-\sum_{i} w_{i}$.

See also [6, (B34)Theorem, Appendix B].

The proof of Theorem 1.2, which is our final goal, is immediate from Theorems 5.3 and 5.4.

\subsection{Numerical examples}

Consider $f=z_{1}^{6}+z_{2}^{6}+z_{3}^{6}+z_{4}^{2}$, a polynomial of Brieskorn-Pham type. $f$ is weighted homogeneous, of degree $d=6$ and weights $\mathbf{w}=(1,1,1,3)$.

The link $K_{f}$ is five-dimensional and $b_{2}=b_{3}=21, h^{2,0}=h^{0,2}=1$ and $h^{1,1}=19$. So $b_{+}=2 h^{2,0}=2$ and $b_{-}=19$, and hence we get $b_{2}=2 h^{2,0}+h^{1,1}$ and the contact-signature $\tau=-17$. See $[\mathbf{1 0}]$ for $b^{+}$and $b^{-}$and the contact signature $\tau$ of a compact Sasakian 5-manifold. 
We compute in fact the Betti number via Theorem 4.1, namely the formula of Milnor-Orlik, as

$$
b_{2}=1-\sum_{i} \frac{q_{i}}{\left[q_{i}\right]}+\sum_{i, j} \frac{q_{i} q_{j}}{\left[q_{i}, q_{j}\right]}
$$

whose value is $1-4+24=21$, since $\left(q_{i}\right)=\left(u_{i}\right)=(6,6,6,2)$ with all $v_{i}=1$. On the other hand, we have

$$
\left(\frac{\partial f}{\partial z_{i}}\right)=\left(6 z_{1}^{5}, 6 z_{2}^{5}, 6 z_{3}^{5}, 2 z_{4}\right)
$$

so that the Milnor algebra $\mathbb{M}_{f}$ is $\mathbb{C}\left[z_{1}, z_{2}, z_{3}\right] /\left(z_{1}^{5}, z_{2}^{5}, z_{3}^{5}\right)$. Then, $\left(\mathbb{M}_{f}\right)_{0}$ is onedimensional so $h^{2,0}=1$. The subspace $\left(\mathbb{M}_{f}\right)_{2 d-\sum_{i} w_{i}}=\left(\mathbb{M}_{f}\right)_{16}$ has dimension 19. So, $h^{1,1}=19$.

Another example is $f=z_{1}^{2}+z_{2}^{3}+z_{3}^{3}+z_{4}^{4}+z_{5}^{6}$. Its degree $d$ is 12 and the weights are $(6,4,4,3,2)$. The link in this case is seven-dimensional and, as we see in [13], $b_{3}=b_{4}=2$ and $h^{3,0}=0, h^{2,1}=1$.

Acknowledgements. The author expresses his gratitude to Professor K. Miyajima with whom discussions on $S^{1}$-Sasakian structure were essential for this work. He is also indebted to Professor K. Kawamura for instructing him on topological affairs concerned with the monodromy map in Section 4. The author, moreover, thanks the referee for valuable comments.

\section{REFERENCES}

[1] W. L. Baily. The decomposition for $V$-manifolds. Amer. J. Math. 78 (1956), 862-888.

[2] D. Blair. Contact Manifolds in Riemannian Geometry (Lecture Notes in Mathematics, 509). Springer-Verlag, 1976.

[3] D. Blair. Riemannian Geometry of Contact and Symplectic Manifolds. Birkhäuser, 2001

[4] W. M. Boothby and H. C. Wang. On contact manifolds. Ann. Math. 68(1958), 721-734.

[5] G. E. Bredon. Introduction to Compact Transformation Groups. Academic Press, New York, 1972.

[6] A. Dimca. Singularities and Topology of Hypersurfaces (Universitext). Springer-Verlag, 1992.

[7] B. Dubrovin. Geometry of 2D topological field theories. Integral Systems and Quantum Groups (Lecture Notes in Mathematics, 1620). Springer-Verlag, 1993, pp. 120-348.

[8] P. Griffiths and J. Harris. Principles of Algebraic Geometry. John Wiley \& Sons, New York, 1978.

[9] Y. Hatakeyama. Some notes on differentiable manifolds with contact structures. Tôhoku Math. J. 15 (1963), 176-181.

[10] M. Itoh. Global geometry of Sasakian manifolds. Proc. Fourth Int. Workshop on Differential Geometry. Ed. Y.-J. Suh. Kyungpook University, Korea, Vol. 4. 2000, pp. 1-7. 
[11] M. Itoh. Contact metric 5-manifolds, CR twistor spaces and integrability. J. Math. Phys. 43 (2002), 3783-3797.

[12] M. Itoh. Contact metric 5-manifolds, CR twistor spaces and integrability. J. Math. Phys. 44 (2003), 366-368 (Erratum).

[13] M. Itoh. Weighted homogeneous polynomial CR manifolds and Tanaka-Hodge numbers. Proc. Hayama Symp. 2002 on Complex Analysis in Several Variables. Ed. K. Miyajima. Shonan Village, 2003, pp. 148-156.

[14] D. McDuff and D. Salamon. Introduction to Symplectic Topology (2nd edn). Clarendon Press, Oxford, 1998.

[15] J. Milnor. Singular Points of Complex Hypersurfaces (Annals Mathematical Studies, 61). Princeton University Press, 1968.

[16] J. Milnor and P. Orlik. Isolated singularities defined by weighted homogeneous polynomials. Topology 9 (1970), 385-393.

[17] J. Morrow and K. Kodaira. Complex Manifolds. Holt, Rinehart and Winston Inc., New York, 1971.

[18] P. Orlik. On the Homology of Weighted Homogeneous Manifolds (Lecture Notes in Mathematics, 298). Springer-Verlag, 1972, pp. 260-269.

[19] S. Sasaki and C.-J. Hsu. On a property of Brieskorn manifolds. Tôhoku Math. J. 28 (1976), 67-78.

[20] J. Steenbrink. Intersection form for quasi-homogeneous singularities. Compositio Math. 34 (1977), 211-223.

[21] J. Steenbrink. Mixed Hodge structures associated with isolated singularities. Proc. Symp. Pure Math. 40 (1983), Part 2, 513-536.

[22] S. Tachibana. On harmonic tensors in compact Sasakian spaces. Tôhoku Math. J. 17 (1965), 271284.

[23] N. Tanaka. A Differential Geometric Study on Strongly Pseudo-Convex Manifolds (Lectures in Mathematics, 9). Kinokuniya, 1975.

[24] S. Tanno. A theorem on regular vector fields and its applications to almost contact structure. Tôhoku Math. J. 17 (1965), 235-238.

[25] C. B. Thomas. Almost regular contact manifolds. J. Diff. Geom. 11 (1976), 521-533.

[26] H. Urakawa. Yang-Mills connections over compact strongly pseudoconvex CR manifolds. Math. Z. 216 (1994), 541-573.

\author{
Mitsuhiro Itoh \\ Institute of Mathematics \\ University of Tsukuba \\ Tsukuba, Japan, 305-8571 \\ (Email: itohm@sakura.cc.tsukuba.ac.jp)
}

